\title{
Quantitative 7-Tesla Imaging of Cortical Myelin Changes in Early Multiple Sclerosis
}

\author{
Valeria Barletta ${ }^{1,2}$, Elena Herranz ${ }^{1,2}$, Constantina A. Treaba ${ }^{1,2}$, Ambica Mehndiratta ${ }^{1}$, \\ Russell Ouellette 1,3,4, Gabriel Mangeat ${ }^{5}$, Tobias Granberg ${ }^{3,4}$, Jacob A. Sloane ${ }^{2,6}$, \\ Eric C. Klawiter ${ }^{1,2}$, Julien Cohen-Adad ${ }^{5}$ and Caterina Mainero ${ }^{1,2 *}$ \\ ${ }^{1}$ Athinoula A. Martinos Center for Biomedical Imaging, Department of Radiology, Massachusetts General Hospital, Boston, \\ MA, United States, ${ }^{2}$ Harvard Medical School, Harvard University, Boston, MA, United States, ${ }^{3}$ Department of Clinical \\ Neuroscience, Karolinska Institutet, Stockholm, Sweden, ${ }^{4}$ Department of Neuroradiology, Karolinska University Hospital, \\ Stockholm, Sweden, ${ }^{5}$ NeuroPoly Lab, Institute of Biomedical Engineering, Polytechnique Montreal, Montreal, QC, Canada, \\ ${ }^{6}$ Department of Neurology, Beth Israel Deaconess Medical Center, Boston, MA, United States
}

\section{OPEN ACCESS}

Edited by:

Deqiang Qiu,

Emory University, United States

Reviewed by:

Ronald Peeters,

University Hospitals Leuven, Belgium Konstantinos Kalafatakis,

University of loannina, Greece

*Correspondence: Caterina Mainero cmainero@mgh.harvard.edu

Specialty section:

This article was submitted to Applied Neuroimaging, a section of the journal

Frontiers in Neurology

Received: 25 May 2021

Accepted: 16 July 2021 Published: 03 September 2021

Citation:

Barletta V, Herranz E, Treaba CA,

Mehndiratta A, Ouellette $R$,

Mangeat G, Granberg T, Sloane JA,

Klawiter EC, Cohen-Adad J and Mainero C (2021) Quantitative 7-Tesla Imaging of Cortical Myelin Changes in

Early Multiple Sclerosis.

Front. Neurol. 12:714820.

doi: 10.3389/fneur.2021.714820
Cortical demyelination occurs early in multiple sclerosis (MS) and relates to disease outcome. The brain cortex has endogenous propensity for remyelination as proven from histopathology study. In this study, we aimed at characterizing cortical microstructural abnormalities related to myelin content by applying a novel quantitative MRI technique in early MS. A combined myelin estimation (CME) cortical map was obtained from quantitative 7-Tesla (7T) $\mathrm{T}_{2}^{*}$ and $\mathrm{T}_{1}$ acquisitions in 25 patients with early MS and 19 healthy volunteers. Cortical lesions in MS patients were classified based on their myelin content by comparison with CME values in healthy controls as demyelinated, partially demyelinated, or non-demyelinated. At follow-up, we registered changes in cortical lesions as increased, decreased, or stable CME. Vertex-wise analysis compared cortical CME in the normal-appearing cortex in $25 \mathrm{MS}$ patients vs. 19 healthy controls at baseline and investigated longitudinal changes at 1 year in $10 \mathrm{MS}$ patients. Measurements from the neurite orientation dispersion and density imaging (NODDI) diffusion model were obtained to account for cortical neurite/dendrite loss at baseline and follow-up. Finally, CME maps were correlated with clinical metrics. CME was overall low in cortical lesions ( $p$ $=0.03$ ) and several normal-appearing cortical areas $(p<0.05)$ in the absence of NODDI abnormalities. Individual cortical lesion analysis revealed, however, heterogeneous CME patterns from extensive to partial or absent demyelination. At follow-up, CME overall decreased in cortical lesions and non-lesioned cortex, with few areas showing an increase $(p<0.05)$. Cortical CME maps correlated with processing speed in several areas across the cortex. In conclusion, CME allows detection of cortical microstructural changes related to coexisting demyelination and remyelination since the early phases of MS, and shows to be more sensitive than NODDI and relates to cognitive performance.

Keywords: quantitative MRI, cortical demyelination, cortical remyelination, NODDI, early multiple sclerosis 


\section{INTRODUCTION}

Demyelinating cortical lesions are a pathologic hallmark of multiple sclerosis (MS), which can develop from the earliest disease stages and relate to disease progression (1-6).

Based on the presence of myelin degradation products inside macrophages and microglia, cortical lesions can be distinguished as actively demyelinating and postdemyelinating $(4,7)$. Ongoing demyelination can coexist within the same lesion with massive remyelination (8); the efficiency of the latter seemingly changes according to disease type and anatomical location $(9,10)$.

Ex vivo MS examinations show that remyelination is more effective in cortical than in white matter (WM) lesions, possibly due to higher availability of oligodendrocytes and their precursors $(10,11)$. Cortical remyelination occurs with a heterogeneous pattern, ranging from extensive ( $\sim 18 \%$ of lesions) to mostly peripheral in the majority of lesions (10). Pathology studies, however, are skewed toward late MS cases, often with associated comorbidities. Knowledge on remyelinating phenomena in the early disease is, thus, very limited, such as the actual clinical impact of cortical demyelination and remyelination in MS patients.

The combined myelin estimation (CME) model extracts the shared information related to myelin content from different quantitative and semiquantitative MRI contrasts, limiting confounding factors such as iron, cortical thickness, and $\mathrm{B}_{0}$ inhomogeneity (12). CME maps obtained from 7-Tesla (7T) quantitative $\mathrm{T}_{2}^{*}$ and 3 -Tesla (3T) magnetization transfer ratio in healthy individuals have previously shown spatial distribution similar to that from histological work stained for myelin (13). More recently, CME obtained from quantitative 7T ultra highresolution $T_{2}^{*}$ and $T_{1}$ maps has been proven to have excellent scan-rescan reproducibility and increased sensitivity to cortical MS pathology compared to individual maps (14).

We hypothesized that cortical microstructural alterations, likely related to myelin content abnormalities, could be measured in vivo using CME from $7 \mathrm{~T}$ quantitative $\mathrm{T}_{2}^{*}$ and $\mathrm{T}_{1}$ mapping in a cohort of 25 early $\mathrm{MS}$ cases relative to 19 age-matched healthy volunteers in both lesioned and normalappearing cortex and would evolve dynamically, suggesting cortical demyelination and remyelination phenomena. As myelin content could be influenced by additional pathological factors including neurite/dendrite loss, measurements from the neurite orientation dispersion and density imaging (NODDI) diffusion model (15-17) were obtained along CME in cortical lesions and whole cortex. Secondarily, the relevance of CME abnormalities on clinical and radiological estimates of disease burden was investigated. Finally, to further validate the CME technique, we assessed the relation between CME values in the healthy subjects and ex vivo myelin density optical measurements (18) across several Brodmann areas.

\section{MATERIALS AND METHODS}

\section{Subjects and Study Procedures}

The Institutional Review Board approved this prospective study, and all subjects gave written informed consent to participate. The work described here has been carried out in accordance with the Declaration of Helsinki.

Twenty-five early MS patients and 19 age-matched healthy volunteers were enrolled between Match 2014 and March 2019. General inclusion criteria were the following: age 1860 , no significant medical history (other than MS for patients), and no MRI contraindications. Inclusion criteria for MS were relapsing-remitting MS diagnosis according to the McDonald criteria (19), disease duration $\leq 5$ years, stable disease-modifying treatment or no treatment and no relapses in the 3 months prior to enrolment, and no corticosteroid use for 1 month prior to enrolment.

At baseline and follow-up, within a week from MRI, patients underwent neurological examination with assessment of the Expanded Disability Status Scale (EDSS) score and Symbol Digit Modalities Test (SDMT). One patient could not perform SDMT because of severe visual impairment. SDMT raw scores were converted to Z-scores (SDMT-z) after correcting for age and education (20).

All subjects were scanned on a 7T MRI whole-body scanner (MAGNETOM, Siemens Healthcare, Erlangen, Germany) equipped with a 32-channel receive head coil, and on a 3T whole-body MRI scanner (MAGNETOM Skyra CONNECTOM, Siemens Healthcare, Erlangen, Germany) using a 64-channel head coil within 1 week from the $7 \mathrm{~T}$ scan. Ten MS patients (mean age, $36 \pm 7.6$ years; females: 8 ) were rescanned with the same protocols after 1 year (mean follow-up time, $1.0 \pm 0.2$ years). Detailed MRI protocol is reported in Table 1. Figure 1 summarizes the imaging procedures.

\section{Cortical Surface Reconstruction}

Cortical surface reconstruction and cortical thickness measurements were performed on the 3T MPRAGE using FreeSurfer v5.3.0 (http://freesurfer.net) after correction for gradient non-linearity-induced distortions. Topological surface reconstruction defects caused by lesions were corrected with lesion in-painting on FreeSurfer.

\section{CME and Diffusion Modeling}

Multilinear regressions were performed using predictors of myelin content $\left(\mathrm{T}_{1}\right.$ and $\left.\mathrm{T}_{2}^{*}\right)$ and a confounding covariate (cortical thickness, which can introduce variable amount of partial volume effect). Secondly, a spatial independent component analysis was used to extract the myelin-specific signal shared by the $T_{1}$ and $\mathrm{T}_{2}^{*}$ maps to obtain the CME \% map (14). A detailed mathematical description of the model has been previously published (13).

Diffusion-weighted images were preprocessed as previously detailed (17), and then analyzed using the NODDI toolbox (v. 0.9, University College London, London, UK; http://mig.cs.ucl.ac.uk/ $\mathrm{mig} / \mathrm{mig} /$ index.php/?n=Tutorial.NODDImatlab/) (15) to obtain maps of neurite density index (NDI) and orientation dispersion index (ODI), a measure of orientational coherence or structural integrity. Maps of CME and NODDI were projected to the cortical surface.

Maps of CME were sampled at 25, 50, and 75\% depth. Maps of NDI and ODI were sampled at $50 \%$ depth to minimize 
TABLE 1 | MRI sequence protocol.

\begin{tabular}{|c|c|c|}
\hline Sequence & $\begin{array}{l}\text { Field } \\
\text { strength }\end{array}$ & Parameters \\
\hline $\begin{array}{l}\text { Single-echo two-dimensional (2D) fast low-angle shot } \\
\mathrm{T}_{2}^{\star} \text {-weighted spoiled gradient echo }\end{array}$ & $7 \mathrm{~T}$ & $\begin{array}{l}\text { Voxel size }=0.33 \times 0.33 \times 1 \mathrm{~mm} \text {; repetition time }=1,700 \mathrm{~ms} \text {; echo time }=21.8 \mathrm{~ms} \\
\text { acquisition time }=\sim 7.5 \mathrm{~min} \text { per slab }(2 \text { slabs })\end{array}$ \\
\hline Multi-echo $2 \mathrm{D} \mathrm{T}_{2}^{*}$ gradient echo & $7 \mathrm{~T}$ & $\begin{array}{l}\text { Voxel size }=0.5 \mathrm{~mm} \text { isotropic; repetition time }=3,680 \mathrm{~ms} \text {; echo time }=3.12+3.32^{*}[1 . .6] \mathrm{ms} \text {; } \\
\text { acquisition time }=\sim 10 \mathrm{~min} \text { per slab (2 slabs); number of echoes }=6\end{array}$ \\
\hline $\begin{array}{l}\text { 3D dual magnetization-prepared rapid gradient echo } \\
\text { (MP2RAGE) }\end{array}$ & $7 \mathrm{~T}$ & $\begin{array}{l}\text { Voxel size }=0.75 \mathrm{~mm} \text { isotropic; repetition time }=5,000 \mathrm{~ms} \text {; echo time }=2.93 \mathrm{~ms} \text {; inversion } \\
\text { time }=900-3,200 \mathrm{~ms} \text {; acquisition time }=10 \mathrm{~min} \text {; flip angles }=4^{\circ} \text { and } 5^{\circ}\end{array}$ \\
\hline $\begin{array}{l}\text { 3D } T_{1} \text {-weighted multi-echo magnetization prepared } \\
\text { rapid acquisition gradient echo (MPRAGE) }\end{array}$ & 3T & $\begin{array}{l}\text { Voxel size }=1 \mathrm{~mm} \text { isotropic; repetition time }=2,530 \mathrm{~ms} \text {; echo time }=1.15,3.03,4.89,6.75 \\
\text { acquisition time }=6 \mathrm{~min} \mathrm{Tl}=1,100 \mathrm{~ms}\end{array}$ \\
\hline 2D echo-planar multishell diffusion weighted & 3Т & $\begin{array}{l}\text { Voxel size }=1.5 \mathrm{~mm} \text { isotropic; b-values }=1,000 \text { and } 5,000 \mathrm{~s} / \mathrm{mm}^{2} ; \text { diffusion directions }=64 \\
\text { and } 128 ; \text { acquisition time }=\sim 12 \mathrm{~min} / 22 \mathrm{~min}\end{array}$ \\
\hline
\end{tabular}

partial volume effects (17). Individual surfaces of CME and NODDI metrics were registered to a common template surface in FreeSurfer and smoothed with a full width at half-maximum kernel of 5 and $10 \mathrm{~mm}$, respectively.

\section{Lesion Quantification and Analysis}

Intracortical, leukocortical, and WM lesions were manually segmented on Slicer (version 4.4.0, 2014; http://www.slicer. org) on $\mathrm{T}_{2}^{*}$-weighted single echo images and checked on the corresponding $\mathrm{T}_{1}$ maps from $7 \mathrm{~T}$ MP2RAGE acquisitions by active collaboration of one radiologist and one neurologist (CT and CM). New lesions were identified on a lesion-bylesion basis through agreement. Lesions were identified as focal hyperintensities that extended for at least three voxels and across two consecutive slices (21). Lesion volumes were quantified by FSL using fslstats from the FMRIB Software Library, v. 5.0 (http:// fsl.fmrib.ox.ac.uk/fsl). Masks of normal-appearing cortex were obtained by subtracting the lesion masks from the whole cortex by using fslmaths on FSL.

Values for CME and NODDI were extracted at 50\% cortical depth in cortical lesion masks and normal-appearing cortex. Since the focus of the study was the cortex, CME and NODDI for leukocortical lesions were measured only in the intracortical portion of the mask.

At baseline, myelination status for each cortical lesion was defined based on the percentage of voxels that had CME values $\leq 2 \mathrm{SD}$ of the mean $\mathrm{CME}$ value in the corresponding cortical area in the control group using the Desikan-Killiany atlas in FreeSurfer. This was done to account for differences in myeloarchitecture across the cortex. Based on the percentage of voxels with reduced CME within an individual lesion, cortical lesions were then classified as demyelinated $(\geq 50 \%)$, partially demyelinated (5-49\%), or non-demyelinated $(<5 \%)$. At follow-up, we registered significant changes in CME within each individual lesion as a variation of at least 1 SD from the basal value (22) (increased, decreased, or stable CME).

\section{Statistical Analysis}

Demographic data were compared between patients and controls by Wilcoxon test. Multilinear regression with age and gender as nuisance factors was used to compare cortical thickness in patients vs. healthy volunteers. CME measures in the healthy cohort were compared by Pearson's correlation to optic myelin measurements previously performed histologically $(13,18)$.

Matched paired $t$-test was used to assess (i) CME and NODDI in cortical lesions vs. normal-appearing homologous contralateral gray matter, (ii) changes in cortical lesion CME and NODDI at follow-up, (iii) changes in cortical lesion and WM lesion volume at follow-up, and (iv) changes in EDSS and SDMT$\mathrm{z}$ at follow-up. A multilinear regression, including age as a nuisance factor, was used to compare CME in cortical lesions vs. cortical gray matter in healthy controls.

Nominal logistic regression was used to correlate CME status at baseline (demyelinated, partially demyelinated, or non-demyelinated) to NODDI continuous values and to correlate CME status at follow-up (increased, decreased, or stable) with lesion type, while multilinear regression was applied to compare NODDI at baseline and follow-up in individual lesions divided based on CME status at follow-up.

To correlate changes in CME with changes in clinical metrics at follow-up, the variation in CME, EDSS, and SDMT$\mathrm{Z}$ was assessed (follow-up-baseline value) and related through Pearson's correlation.

Spearman's correlation was used to relate cortical CME to WML and cortical lesion load.

A vertex-wise general linear model (GLM) on FreeSurfer was used to (i) assess differences in cortical CME and NODDI between patients and controls, (ii) assess longitudinal CME changes in patients, (iii) correlate cortical CME with EDSS and SDMT-z, and (iv) correlate cortical thickness with EDSS and SDMT-z. In all GLM analyses, a cluster-wise correction for multiple comparisons using Monte Carlo simulation with 10,000 iterations was applied. Significant clusters were localized using the Desikan-Killiany atlas in FreeSurfer. Since the cortical thickness map was used to construct the CME cortical map, the vertex-wise between the two metrics was not investigated, but cortical thickness was included in all CME GLM analyses as a vertex-wise covariate of no interest.

Statistical analysis was performed on JMP pro v13. A $p<0.05$ was considered significant. 


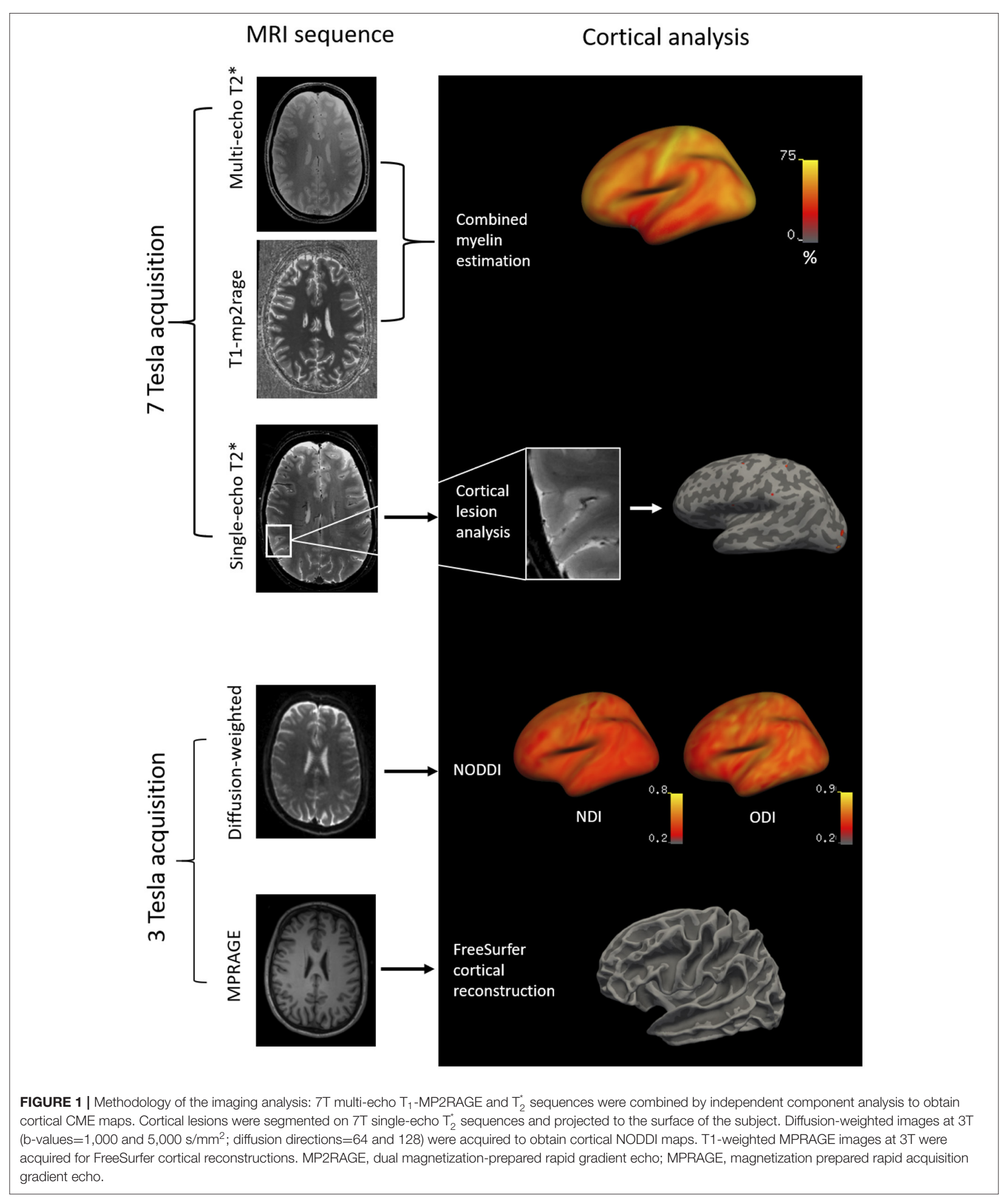


TABLE 2 | Demographic, clinical and radiological characteristics of the study subjects.

\begin{tabular}{|c|c|c|c|}
\hline Characteristic & $\begin{array}{l}\text { Healthy controls } \\
\qquad n=19\end{array}$ & $\begin{array}{c}\text { MS patients } \\
\quad n=25\end{array}$ & $p$ \\
\hline Age, mean $\pm S D$ & $35.8 \pm 10.2$ & $38.2 \pm 8.9$ & $0.3^{\mathrm{a}}$ \\
\hline Disease duration in years, median (range) & - & $1.9(0.6-4.7)$ & - \\
\hline Age at disease onset, mean \pm SD & - & $35 \pm 8.1$ & - \\
\hline Disease modifying treatment $n$ & - & 22 & - \\
\hline - Dimethyl fumarate $n$ & - & 10 & - \\
\hline - Glatiramer acetate $n$ & - & 5 & - \\
\hline - Interferon beta-1a $n$ & - & 4 & - \\
\hline - Natalizumab $n$ & - & 2 & - \\
\hline Intracortical lesion volume $\mathrm{mm}^{3}$, mean $\pm \mathrm{SD}$ & - & $162 \pm 161$ & - \\
\hline Leukocortical lesion volume $\mathrm{mm}^{3}$, mean $\pm \mathrm{SD}$ & - & $164 \pm 180$ & - \\
\hline WM lesion volume $\mathrm{mm}^{3}$, mean $\pm \mathrm{SD}$ & - & $1,357 \pm 2160$ & - \\
\hline
\end{tabular}

$S D$, standard deviation.

${ }^{a}$ By Wilcoxon test.

${ }^{b}$ By Fisher's exact test.

${ }^{c}$ By multilinear regression correcting for age and gender.

\section{RESULTS}

\section{Demographic, Clinical, and MRI Results}

The demographic, clinical, and MRI characteristics of the study subjects are summarized in Table 2.

No lesions were detected in the healthy group. At baseline, 103 cortical lesions were detected in 20 of 25 (80\%) MS patients: 62 (60\%) intracortical, 41 (40\%) leukocortical (Table 2, Figures 2A-F).

At 1-year follow-up, 25 new cortical lesions (23 intracortical and 2 leukocortical) developed in 4 of 10 patients. Overall, both cortical and WM lesion volumes tended to be higher at followup, although not significantly. Cortical thickness did not change significantly (Table 3).

Diffusion NODDI images were acquired at baseline in $21 \mathrm{MS}$ patients (18 of which showed cortical lesions) and 17 healthy controls, and in 9 of 10 patients rescanned at 1-year follow-up.

\section{CME and NODDI Metrics Along the Normal-Appearing Cortex}

In healthy subjects, we found a positive correlation between CME at each cortical depth and myelin density optical measurements previously obtained in the corresponding cortical regions (13, 18) of a healthy adult human brain (by Pearson's correlation; Figure 3).

At baseline, the GLM analysis revealed several clusters of significantly reduced CME in MS patients compared to the control group in the normal-appearing cortex at all cortical depths (Figure 4A). Table 4 summarizes the localization of the clusters in which CME values were significantly different in MS patients vs. controls. No differences in NDI or ODI were seen at GLM analysis between patients and controls along the whole cortex.

In 10 patients rescanned at the 1-year follow-up, CME \% was overall reduced in normal-appearing cortex $(50.0 \pm 2$ vs. $49.7 \pm 2, p=0.02$ by matched pairs $t$-test). The vertex-wise comparison showed, however, fewer clusters with increase in CME at follow-up (Figure 4B).

\section{CME and NODDI Metrics in Cortical Lesions}

Overall, cortical lesions showed lower mean CME values relative to the contralateral normal-appearing cortex $(p=0.04$ by $t$ test) and to cortex of healthy subjects $(p=0.03$ by multilinear regression), while no differences were detected for either NDI or ODI in the same regions (Table 5).

Individual cortical lesion analysis showed that 62 of $103(60 \%)$ of them were demyelinated, either extensively $(33 / 103,32 \%)$ or partially $(29 / 103,28 \%)$. The percentage of demyelination did not relate to lesion type $(p=0.4$ by Wilcoxon test for intracortical vs. leukocortical). Forty-one out of 103 lesions showed $<5 \%$ demyelinated voxels.

Values of NDI and ODI were extracted individually for 97 cortical lesions (18 MS subjects) at baseline. No difference was seen in either NDI or ODI in MS cortical lesions relative to the contralateral normal-appearing cortex in patients (respectively, $p=0.4$ and 0.6 , by $t$-test) and to the cortex of healthy controls 

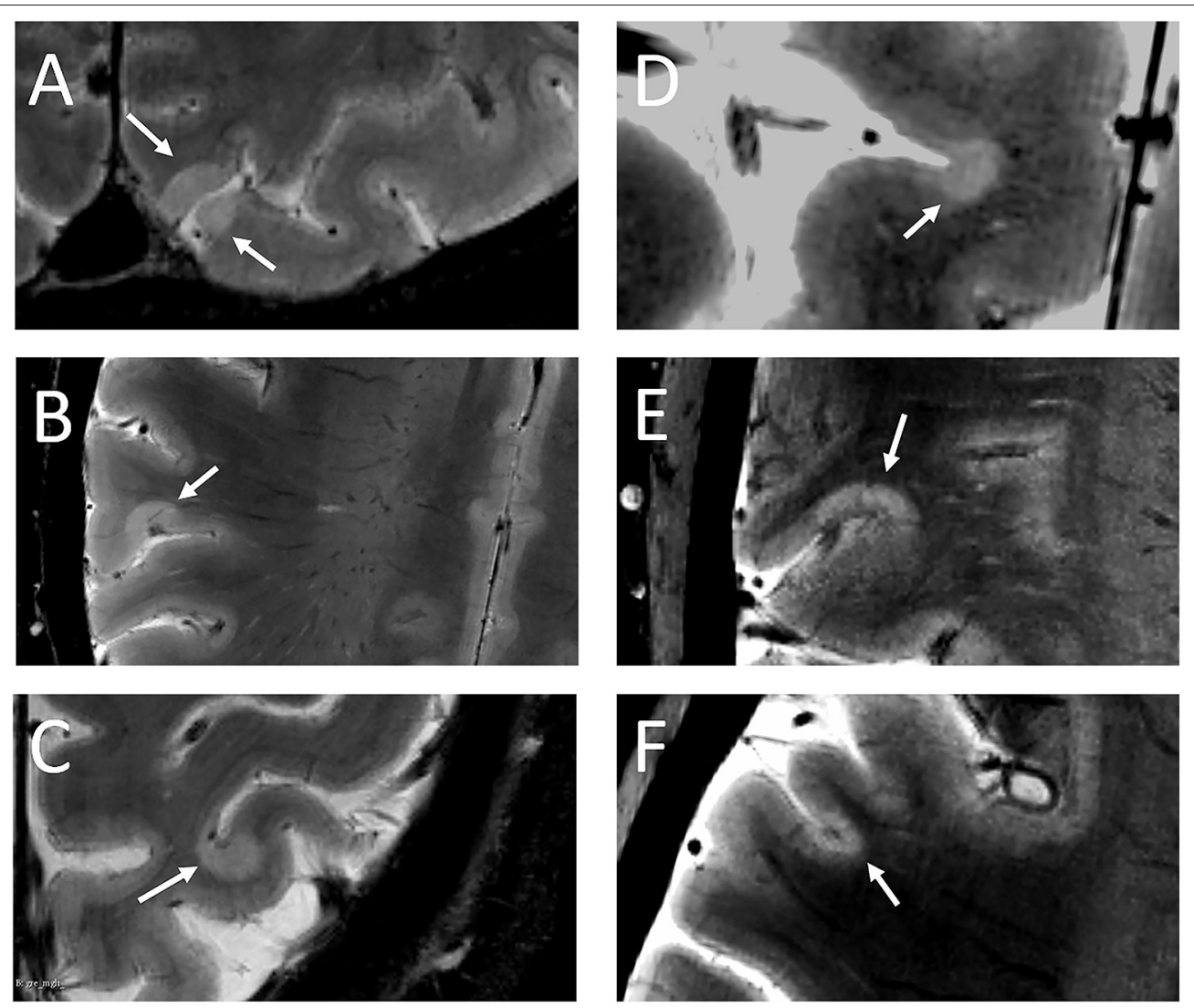

FIGURE 2 | White arrows indicate leukocortical (A-C) and intracortical (D-F) lesions detected in the MS cohort.

TABLE 3 | MRI characteristics of the MS patients rescanned after 1 year.

\begin{tabular}{lccc}
\hline MRI characteristic & Baseline & Follow-up & $\boldsymbol{p}$ \\
\hline $\begin{array}{l}\text { Cortical thickness mm, } \\
\text { mean } \pm \mathrm{SD}\end{array}$ & $2.40 \pm 0.08$ & $2.41 \pm 0.07$ & $0.67^{\mathrm{a}}$ \\
$\begin{array}{l}\text { Total cortical lesion count, } \\
\text { median (range) }\end{array}$ & $5(1-22)$ & $5.5(1-33)$ & - \\
$\begin{array}{l}\text { WM lesion volume } \mathrm{mm}^{3}, \\
\text { mean } \pm \mathrm{SD}\end{array}$ & $1,350 \pm 2,162$ & $1,472 \pm 2,025$ & $0.3^{\mathrm{a}}$ \\
$\begin{array}{l}\text { Total cortical lesion volume } \\
\mathrm{mm}^{3}, \text { mean } \pm \mathrm{SD}\end{array}$ & $253 \pm 242$ & $369 \pm 566$ & $0.4^{\mathrm{a}}$ \\
\end{tabular}

$S D$, standard deviation.

${ }^{a}$ By matched pairs t-test.

( $p=0.3$ and 0.2 by multilinear regression) (Table 5). Values of NDI and ODI were also grouped based on cortical lesion myelination status as measured by CME and did not show any difference between demyelinated, partially demyelinated, or non-demyelinated cortical plaques (for NDI, $p=0.6$; for ODI, $p=0.7$, by nominal logistic regression; subject ID was an adjusting factor).

At follow-up, cortical lesions showed an overall reduction in CME \% ( $48.8 \pm 7.2$ vs. $46.5 \pm 6.1, p=0.03$ by $t$-test), suggesting further global demyelination.
Individual CME lesion analysis revealed that out of the total 63 lesions reexamined at follow-up, 26 (41\%) showed reduction in their basal CME values $>1$ SD, 27 (43\%) showed no significant changes, while $10(16 \%)$ registered an increase $\geq 1$ SD of CME basal values. Interestingly, two of the lesions with increased CME were fully demyelinated at baseline but reached mean CME values in the range of normality at follow-up, suggesting effective remyelination.

Lesion type (intracortical or leukocortical) was not a predictor of CME changes at follow up $(p=0.59$ by nominal logistic regression).

No significant changes were seen in NODDI metrics in cortical lesions (by paired $t$-test) at follow-up. However, when individual cortical lesions were divided based on CME changes at follow-up (increased, stable, or decreased), a significant increase was seen in ODI in lesions with increased CME (ODI mean \pm $\mathrm{SD}, 0.49 \pm 0.08$ at baseline vs. $0.57 \pm 0.02$ at follow-up, $p=$ 0.009 by multilinear regression; subject ID was included as a confounding factor).

\section{Correlations With Clinical and MRI Disease Burden}

Vertex-wise positive correlations were found between cortical CME and SDMT-z (Figure 5), while no clusters were found for EDSS. For SDMT-z, positive correlations with myelin content 


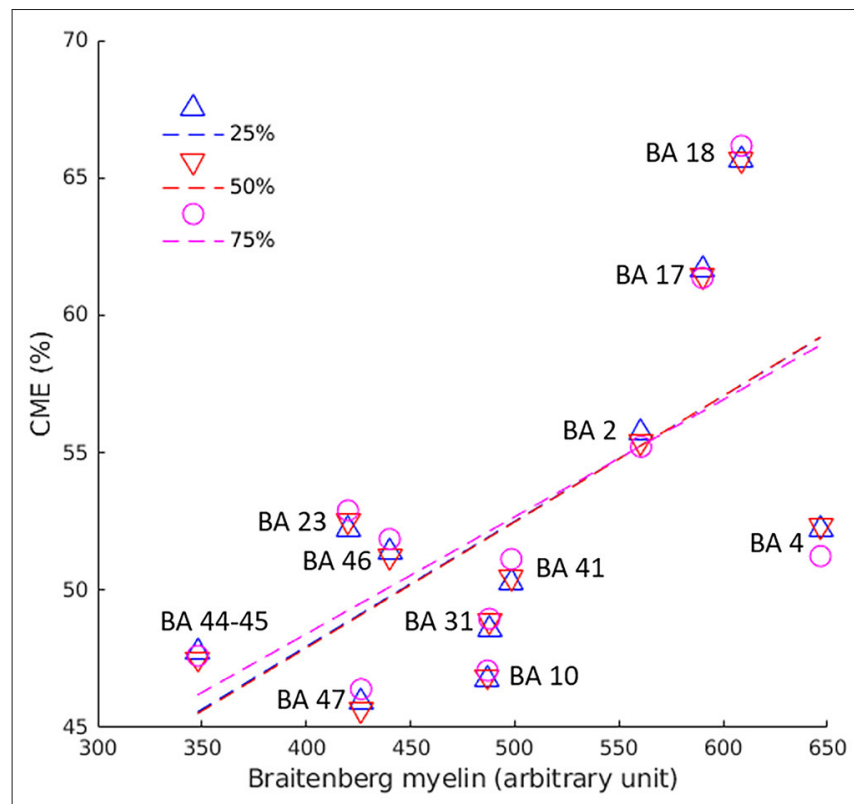

FIGURE 3 | Pearson's correlation between CME values in the cortex and myelin optic measurements across 12 BA of healthy controls. Pearson's coefficient was $0.6(p=0.036)$ at $25 \%$ (blue triangles/line), $0.7(p=0.022)$ at $50 \%$ (red triangles/line), and $0.7(p=0.024)$ at $75 \%$ (pink circles/line) of cortical depth from the pial surface. BA, Broadmann area.

were found mostly at the mid-cortical level in both hemispheres. In the right hemisphere, most clusters were found in the superior and inferior parietal, lateral occipital, and postcentral cortex. In the left hemisphere, clusters were found in the rostral middle frontal, superior frontal, superior parietal, and posterior cingulate cortex. Table 6 indicates the localization of clusters of correlation between CME and SDMT-z at three cortical depths.

Neither EDSS nor SDMT-z registered significant changes at follow-up (for EDSS (mean \pm SD): at baseline $1.7 \pm 0.8$, at followup $1.7 \pm 0.7, p=0.8$; for SDMT-z (mean \pm SD): at baseline 0.9 \pm 1.1 , at follow-up $0.7 \pm 1.3, p=0.5$ by matched pair $t$-test). Percentage changes in lesion CME did not correlate with changes in EDSS or SDMT-z (respectively, $p=0.3$ and 0.9 , by Pearson's).

No correlations were found between cortical thickness and either EDSS or SDMT-z. There was no correlation between CME and WM or cortical lesion volume.

\section{DISCUSSION}

In this study, we applied CME from quantitative $7 \mathrm{~T}$ acquisitions to assess microstructural changes likely related to myelin content in cortical lesions and normal-appearing cortex of early MS cases. To account for the possible influence of neural loss on CME, NODDI measurements were also obtained. In patients, mean CME was overall abnormally decreased in cortical lesions and several regions of the cortical mantle, without significant changes in diffusion metrics or cortical thinning, suggesting early cortical demyelination in the absence of decreased neuroaxonal density and overt cortical atrophy. Individual

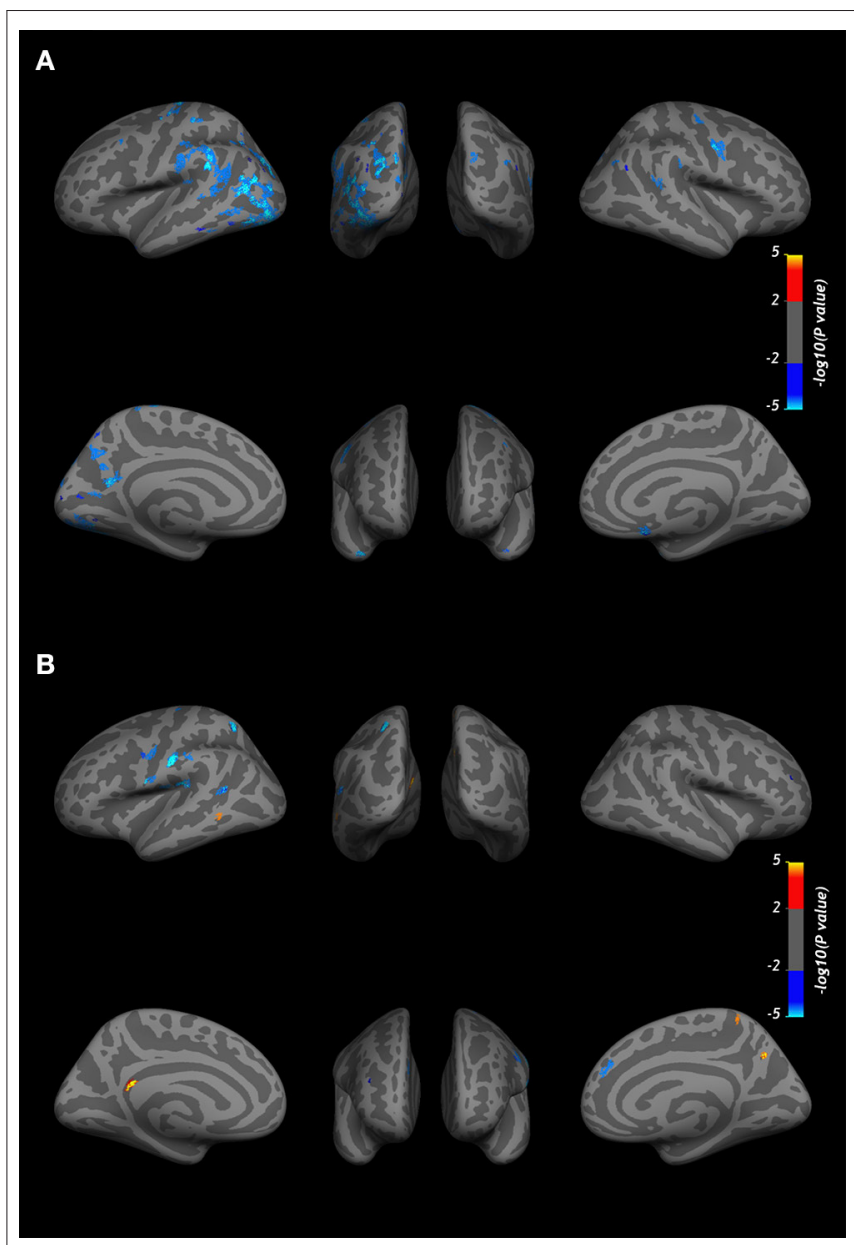

FIGURE 4 | Overlay of the GLM significance map $(p<0.05$ corrected for multiple comparisons). (A) Clusters of significantly reduced CME in the normal-appearing cortex of MS patients vs. healthy controls. (B) Variation in CME values in the normal-appearing cortex of $10 \mathrm{MS}$ patients rescanned at 1-year follow-up. Blue clusters indicate areas of CME reduction, and yellow clusters indicate CME increase. Both analyses were performed on normal-appearing cortex at three cortical depths (25, 50, and $75 \%)$ from the pial surface, overlapped in the figure. Color bars show -log10(p-value).

cortical lesion analysis, however, revealed heterogeneous CME patterns, possibly reflecting underlying concomitant phenomena of demyelination, incomplete demyelination, or remyelination.

Cortical CME in the control group correlated across several Brodmann areas with myelin optical measurements obtained from histology (18), providing indirect evidence that the metric is related to cortical myelin content. Compared to previous CME data obtained by combining $7 \mathrm{~T}_{2}^{*}$ with $3 \mathrm{~T}$ magnetization transfer ratio (13), the current approach enables acquisition of higher-resolution images, which is more effective for laminar cortical pathology analysis.

Cortical lesions were present in $80 \%$ of MS subjects, confirming their high detection in early MS in vivo by 7T MRI $(17,23,24)$.

Overall CME was reduced both in cortical lesions and normal-appearing cortex, suggesting the presence of widespread 
TABLE 4 | Localization of clusters indicating lower cortical CME in MS patients vs. healthy subjects at three cortical depths.

\section{Left hemisphere}

$25 \%$ from pial surface

$50 \%$ from pial surface

75\% from pial surface

\section{Right hemisphere}

$25 \%$ from pial surface

$50 \%$ from pial surface

$75 \%$ from pial surface

\section{Fusiform, precentral}

Lateral occipital (387 mm²), inferior parietal (282 mm²), superior parietal (179 $\left.\mathrm{mm}^{2}\right)$, supramarginal $\left(119 \mathrm{~mm}^{2}\right)$, precentral, precuneus, fusiform, postcentral, temporal pole, lingual gyrus

Lingual gyrus (1882 mm²), supramarginal (962 mm²), superior parietal (390 mm²), inferior parietal (337 mm²), precentral (309 mm²), precuneus $\left(281 \mathrm{~mm}^{2}\right)$, peri-calcarine $\left(186 \mathrm{~mm}^{2}\right)$, inferior temporal $\left(157 \mathrm{~mm}^{2}\right)$, banks of the superior temporal sulcus (124 mm²), postcentral, cuneus, paracentral, caudal middle frontal, middle temporal, superior frontal

Temporal pole, precentral

Temporal pole, medial orbito-frontal, fusiform

Precentral (307 mm²), superior parietal, banks of the superior temporal sulcus, inferior parietal, caudal anterior cingulate, fusiform, supramarginal, temporal pole

Total area is indicated in brackets for clusters with area $\geq 100 \mathrm{~mm}^{2}$.

TABLE 5 | Results from the CME and neurite orientation dispersion and density index modeling in the cortex of early stage MS subjects and healthy controls.

\begin{tabular}{|c|c|c|c|c|c|c|}
\hline Metric & MS cortical lesions & MS contralateral normal appearing cortex & $p$ & MS whole cortex & Healthy controls whole cortex & $p$ \\
\hline $\mathrm{CME} \%$ mean $\pm \mathrm{SD}$ & $47.1 \pm 6.4$ & $50.0 \pm 8.6$ & $0.04^{a}$ & $50.2 \pm 1.8$ & $50.6 \pm 1.8$ & $0.4^{b}$ \\
\hline $\mathrm{NDI}$ mean $\pm \mathrm{SD}$ & $0.42 \pm 0.03$ & $0.42 \pm 0.03$ & $0.4^{a}$ & $0.43 \pm 0.01$ & $0.43 \pm 0.02$ & $0.8^{\mathrm{b}}$ \\
\hline ODI mean \pm SD & $0.55 \pm 0.04$ & $0.56 \pm 0.05$ & $0.6^{\mathrm{a}}$ & $0.56 \pm 0.01$ & $0.56 \pm 0.01$ & $1.0^{\mathrm{b}}$ \\
\hline
\end{tabular}

${ }^{a}$ By matched pairs t-test.

${ }^{b}$ By multilinear regression (adjusted for age).

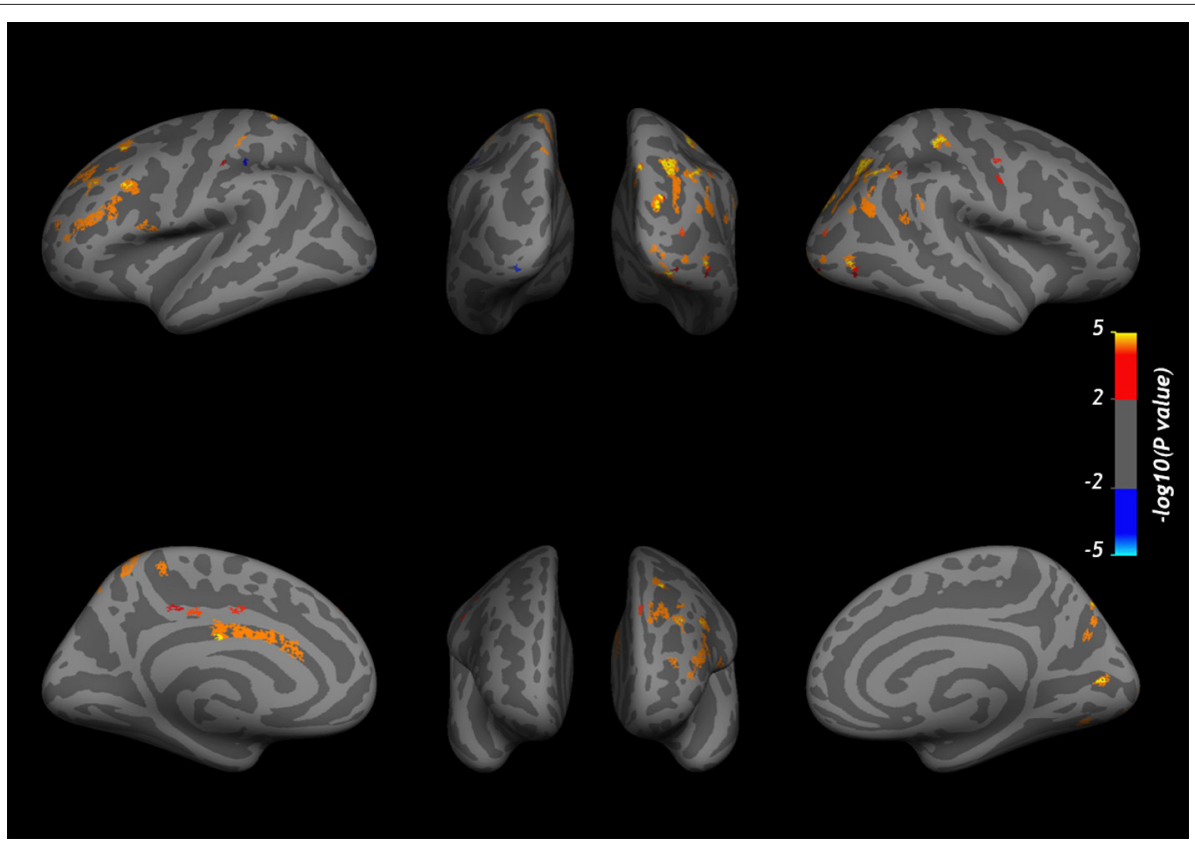

FIGURE 5 | Overlay of the GLM significance map ( $p<0.05$ corrected for multiple comparisons) showing vertex-wise positive correlations between CME and SDMT-z at three cortical depths in MS patients. Color bar shows -log10 ( $\mathrm{p}$-value). Cortical thickness and WM lesion load were included in the analysis as adjusting factors.

microstructural abnormalities related to demyelination. When we performed an individual cortical lesion analysis, we found that $60 \%$ of cortical lesions had some degree of demyelination, while the remaining had reduced CME in $<5 \%$ of the total voxels. This pattern is supported by previous combined histopathological/MRI analyses of autoptic cortical MS tissue, which showed that some of the cortical hyperintensities detected by $7 \mathrm{~T} \mathrm{~T}_{2}^{*}$ sequences $(7 / 83,8 \%)$ represented incompletely demyelinated or remyelinating areas (24). 
TABLE 6 | Localization of clusters indicating positive correlation between cortical CME and SDMT z-scores.

\begin{tabular}{ll}
\hline $\begin{array}{l}\text { Left hemisphere } \\
25 \% \text { from pial surface }\end{array}$ & $\begin{array}{l}\text { Caudal middle frontal, pars opercularis, rostral middle frontal, postcentral, superior parietal, superior frontal, supramarginal, posterior } \\
\text { cingulate }\end{array}$ \\
$\begin{array}{l}\text { Rostral middle frontal }\left(384 \mathrm{~mm}^{2}\right) \text {, superior frontal }\left(285 \mathrm{~mm}^{2}\right) \text {, superior parietal }\left(281 \mathrm{~mm}^{2}\right), \text { posterior cingulate }\left(236 \mathrm{~mm}^{2}\right), \text { caudal } \\
\text { middle frontal }\left(135 \mathrm{~mm}^{2}\right), \text { caudal anterior cingulate, para-central, pars opercularis, lateral occipital, isthmus cingulate }\end{array}$ \\
$\begin{array}{l}\text { None } \\
75 \% \text { from pial surface } \\
\text { Right hemisphere } \\
25 \% \text { from pial surface }\end{array}$ \\
$\begin{array}{l}\text { Superior parietal, postcentral, inferior parietal, precentral, superior frontal, peri-calcarine } \\
\text { Sum pial surface }\end{array}$ & $\begin{array}{l}\text { Superior parietal }\left(446 \mathrm{~mm}^{2}\right) \text {, inferior parietal }\left(365 \mathrm{~mm}^{2}\right) \text {, lateral occipital }\left(364 \mathrm{~mm}^{2}\right), \text { postcentral }\left(120 \mathrm{~mm}^{2}\right), \text { precuneus, peri-calcarine, } \\
\text { lingual, fusiform, supramarginal, precentral }\end{array}$ \\
$75 \%$ from pial surface & Inferior parietal $\left(125 \mathrm{~mm}^{2}\right)$, lateral occipital $\left(100 \mathrm{~mm}^{2}\right)$, superior parietal, superior temporal
\end{tabular}

Total area is indicated in brackets for clusters with area $\geq 100 \mathrm{~mm}^{2}$.

Despite an overall decrease in lesion CME at the 1-year followup, significant increase in few cortical lesions and some areas in the normal-appearing cortex was detected, suggesting the presence of both demyelination and remyelination processes, as observed in histopathology studies $(9-11,25)$.

The results from the NODDI analysis showed that neither NDI nor ODI was altered in early MS patients, while CME showed significant differences both in cortical lesions and in normal-appearing cortex, proving to be very sensitive in disclosing early microstructural damage.

The NDI relates to neuroaxonal density and has been found to be reduced in the MS WM and cortex, especially in the progressive disease $(16,17,26)$. In ex vivo studies, NDI has also been found to correlate with myelin content $(16,27)$. Our results suggest that, at least in our cohort, cortical demyelination is not accompanied by neural loss in the early phase of the disease and that CME seems sensitive in detecting early cortical myelin changes. Additionally, relative to NODDI, the CME approach from 7T MRI enables acquisition of higher-resolution images, which may be more effective for laminar cortical pathology analysis.

The higher ODI found in cortical lesions with increased CME at follow-up could indicate higher arborization of the dendrites in repairing lesions, as opposed to the lower arborization found in demyelinating lesions $(15,16)$. We cannot completely rule out, however, that the increase in CME observed at follow-up could have been, at least in part, influenced by resolution of possible inflammatory processes within cortical lesions (28).

A lower information processing speed was associated with lower CME in several regions, especially in the right superior and inferior parietal, lateral occipital, and post-central cortex and in the left rostral middle frontal, superior frontal, superior parietal, and posterior cingulate. These areas are involved in spatial orientation, object recognition, working memory, and executive attention.

No voxel-wise correlations with EDSS were found, probably due to the low scores and scarce variability in the EDSS in our early cohort (mean EDSS \pm SD was $1.8 \pm 1$ ).
Limitations to our study include the small follow-up sample that might have affected the statistical power to investigate further longitudinal changes in cortical CME, as well as the lack of longitudinal data for healthy subjects, although high scanrescan reproducibility of CME in healthy subjects was previously demonstrated (14).

In conclusion, CME from ultra-high-resolution quantitative $\mathrm{T}_{2}^{*}$ and $\mathrm{T}_{1}$ at $7 \mathrm{~T}$ is a sensitive technique for detecting, in early MS, cortical microstructural abnormalities related to myelin changes. This promising technique finds further application in other neurological diseases caused by altered cortical integrity.

\section{DATA AVAILABILITY STATEMENT}

The original contributions presented in the study are included in the article/supplementary material, further inquiries can be directed to the corresponding author/s.

\section{ETHICS STATEMENT}

The studies involving human participants were reviewed and approved by Mass General Brigham Institutional Review Board. The patients/participants provided their written informed consent to participate in this study.

\section{AUTHOR CONTRIBUTIONS}

CM and VB contributed to conception and design of the study. VB performed statistical analysis and wrote the first draft of the manuscript. VB, EH, CT, AM, and RO contributed to data acquisition and analysis. All authors contributed to manuscript revision, read, and approved the submitted version.

\section{FUNDING}

This work was supported partly by the National Multiple Sclerosis Society (NMSS RG-1802-30468) and the National Institute of Health (NIH R01NS078832201 A1). 


\section{REFERENCES}

1. Peterson JW, Bö L, Mörk S, Chang A, Trapp BD. Transected neurites, apoptotic neurons, and reduced inflammation in cortical multiple sclerosis lesions. Ann Neurol. (2001) 50:389-400. doi: 10.1002/ana.1123

2. Calabrese M, De Stefano N, Atzori M, Bernardi V, Mattisi I, Barachino L, et al. Detection of cortical inflammatory lesions by double inversion recovery magnetic resonance imaging in patients with multiple sclerosis. Arch Neurol. (2007) 64:1416-22. doi: 10.1001/archneur.64.10.1416

3. Calabrese M, Filippi M, Gallo P. Cortical lesions in multiple sclerosis. Nat Rev Neurol. (2010) 6:438-44. doi: 10.1038/nrneurol.2010.93

4. Lucchinetti CF, Popescu BF, Bunyan RF, Moll NM, Roemer SF, Lassmann H, et al. Inflammatory cortical demyelination in early multiple sclerosis. $\mathrm{N} \mathrm{Engl} \mathrm{J}$ Med. (2011) 365:2188-97. doi: 10.1056/NEJMoa1100648

5. Mainero C, Louapre C, Govindarajan ST, Giannì C, Nielsen AS, CohenAdad J, et al. A gradient in cortical pathology in multiple sclerosis by in vivo quantitative $7 \mathrm{~T}$ imaging. Brain. (2015) 138(Pt 4):932-45. doi: 10.1093/brain/awv011

6. Treaba CA, Granberg TE, Sormani MP, Herranz E, Ouellette RA, Louapre $\mathrm{C}$, et al. Longitudinal characterization of cortical lesion development and evolution in multiple sclerosis with 7.0-T MRI. Radiology. (2019) 291:740-9. doi: 10.1148/radiol.2019181719

7. Kuhlmann T, Ludwin S, Prat A, Antel J, Brück W, Lassmann H. An updated histological classification system for multiple sclerosis lesions. Acta Neuropathol. (2017) 133:13-24. doi: 10.1007/s00401-016-1653-y

8. Prineas JW, Kwon EE, Cho ES, Sharer LR. Continual breakdown and regeneration of myelin in progressive multiple sclerosis plaques. Ann N Y Acad Sci. (1984) 436:11-32. doi: 10.1111/j.1749-6632.1984.tb14773.x

9. Patrikios P, Stadelmann C, Kutzelnigg A, Rauschka H, Schmidbauer M, Laursen $\mathrm{H}$, et al. Remyelination is extensive in a subset of multiple sclerosis patients. Brain. (2006) 129(Pt 12):3165-72. doi: 10.1093/brain/awl217

10. Albert M, Antel J, Brück W, Stadelmann C. Extensive cortical remyelination in patients with chronic multiple sclerosis. Brain Pathol. (2007) 17:129-38. doi: 10.1111/j.1750-3639.2006.00043.x

11. Strijbis EMM, Kooi EJ, van der Valk P, Geurts JJG. Cortical remyelination is heterogeneous in multiple sclerosis. J Neuropathol Exp Neurol. (2017) 76:390-401. doi: 10.1093/jnen/nlx023

12. Cohen-Adad J, Polimeni JR, Helmer KG, Benner T, McNab JA, Wald LL, et al. $\mathrm{T}_{2}^{*}$ mapping and $\mathrm{B} 0$ orientation-dependence at $7 \mathrm{~T}$ reveal cyto- and myeloarchitecture organization of the human cortex. Neuroimage. (2012) 60:1006-14. doi: 10.1016/j.neuroimage.2012.01.053

13. Mangeat G, Govindarajan ST, Mainero C, Cohen-Adad J. Multivariate combination of magnetization transfer, $\mathrm{T} 2 *$ and B0 orientation to study the myelo-architecture of the in vivo human cortex. Neuroimage. (2015) 119:89-102. doi: 10.1016/j.neuroimage.2015.06.033

14. Mangeat G, Badji A, Ouellette R, Treaba CA, Herranz E, Granberg $\mathrm{T}$, et al. Changes in structural network are associated with cortical demyelination in early multiple sclerosis. Hum Brain Mapp. (2018) 39:213346. doi: 10.1002/hbm.23993

15. Zhang $\mathrm{H}$, Schneider $\mathrm{T}$, Wheeler-Kingshott CAG, Alexander DC. NODDI: Practical in vivo neurite orientation dispersion and density imaging of the human brain. Neuroimage. (2012) 61:1000-16. doi: 10.1016/j.neuroimage.2012.03.072

16. Grussu F, Schneider T, Tur C, Yates RL, Tachrount M, Ianuş A, et al. Neurite dispersion: a new marker of multiple sclerosis spinal cord pathology? Ann Clin Transl Neurol. (2017) 4:663-79. doi: 10.1002/acn3.445

17. Granberg T, Fan Q, Treaba CA, Ouellette R, Herranz E, Mangeat G, et al. In vivo characterization of cortical and white matter neuroaxonal pathology in early multiple sclerosis. Brain. (2017) 140:2912-26. doi: 10.1093/brain/awx247
18. Braitenberg V. A note on myeloarchitectonics. J Comp Neurol. (1962) 118:141-56. doi: 10.1002/cne.901180202

19. Polman CH, Reingold SC, Banwell B, Clanet M, Cohen JA, Filippi M, et al. Diagnostic criteria for multiple sclerosis: 2010 revisions to the McDonald criteria. Ann Neurol. (2011) 69:292-302. doi: 10.1002/ana. 22366

20. Parmenter BA, Testa SM, Schretlen DJ, Weinstock-Guttman B, Benedict RH. The utility of regression-based norms in interpreting the minimal assessment of cognitive function in multiple sclerosis (MACFIMS). J Int Neuropsychol Soc. (2010) 16:6-16. doi: 10.1017/S1355617709990750

21. Louapre C, Govindarajan ST, Giannì C, Langkammer C, Sloane JA, Kinkel RP. Beyond focal cortical lesions in MS: an in vivo quantitative and spatial imaging study at 7T. Neurology. (2015) 85:1702-9. doi: 10.1212/WNL.0000000000002106

22. Bodini B, Veronese M, García-Lorenzo D, Battaglini M, Poirion E, Chardain A, et al. Dynamic imaging of individual remyelination profiles in multiple sclerosis. Ann Neurol. (2016) 79:726-38. doi: 10.1002/ana.24620

23. Pitt D, Boster A, Pei W, Wohleb E, Jasne A, Zachariah CR, et al. Imaging cortical lesions in multiple sclerosis with ultra-high-field magnetic resonance imaging. Arch Neurol. (2010) 67:812-8. doi: 10.1001/archneurol.2010.148

24. Kilsdonk ID, Jonkman LE, Klaver R, van Veluw SJ, Zwanenburg JJ, Kuijer JP, et al. Increased cortical grey matter lesion detection in multiple sclerosis with 7 T MRI: a post-mortem verification study. Brain. (2016) 139(Pt 5):1472-81. doi: 10.1093/brain/aww037

25. Bramow S, Frischer JM, Lassmann H, Koch-Henriksen N, Lucchinetti CF, Sørensen PS, et al. Demyelination versus remyelination in progressive multiple sclerosis. Brain. (2010) 133:2983-98. doi: 10.1093/brain/awq250

26. Rahmanzadeh R, Lu P, Barakovic M, Weigel M, Maggi P, Nguyen TD, et al. Myelin and axon pathology in multiple sclerosis assessed by myelin water and multi-shell diffusion imaging. Brain. (2021) 144:1684-96. doi: 10.1093/brain/awab088

27. Jespersen SN, Bjarkam CR, Nyengaard JR, Chakravarty MM, Hansen $B$, Vosegaard $T$, et al. Neurite density from magnetic resonance diffusion measurements at ultrahigh field: comparison with light microscopy and electron microscopy. Neuroimage. (2010) 49:205-16. doi: 10.1016/j.neuroimage.2009.08.053

28. Herranz E, Giannì C, Louapre C, Treaba CA, Govindarajan ST, Ouellette R, et al. Neuroinflammatory component of gray matter pathology in multiple sclerosis. Ann Neurol. (2016) 80:776-90. doi: 10.1002/ana.24791

Conflict of Interest: The authors declare that the research was conducted in the absence of any commercial or financial relationships that could be construed as a potential conflict of interest.

Publisher's Note: All claims expressed in this article are solely those of the authors and do not necessarily represent those of their affiliated organizations, or those of the publisher, the editors and the reviewers. Any product that may be evaluated in this article, or claim that may be made by its manufacturer, is not guaranteed or endorsed by the publisher.

Copyright (c) 2021 Barletta, Herranz, Treaba, Mehndiratta, Ouellette, Mangeat, Granberg, Sloane, Klawiter, Cohen-Adad and Mainero. This is an open-access article distributed under the terms of the Creative Commons Attribution License (CC BY). The use, distribution or reproduction in other forums is permitted, provided the original author(s) and the copyright owner(s) are credited and that the original publication in this journal is cited, in accordance with accepted academic practice. No use, distribution or reproduction is permitted which does not comply with these terms. 